“C 2013 IEEE. Personal use of this material is permitted. Permission from IEEE must be obtained for all other uses, in any current or future media, including reprinting/republishing this material for advertising or promotional purposes, creating new collective works, for resale or redistribution to servers or lists, or reuse of any copyrighted component of this work in other works." 


\title{
A New Approach to Applying Discrete Sliding Mode Control to 2D Systems
}

\author{
Ahmadreza Argha, Li Li and Steven W. Su
}

\begin{abstract}
Sliding mode control has been applied previously to a specific form of 2D systems (Roesser model). In this paper a new approach (1D vectorial form) is introduced for this problem. Using 1D form to represent 2D systems can be used as an alternative strategy to reduce the inherent complexity of $2 \mathrm{D}$ systems and their applications. Unlike Wave Advanced Model (WAM) form (proposed by Porter and Aravena), the suggested 1D vectorial form, in this paper, has invariable dimension and consequently can be converted to regular form for sliding mode control (SMC). In this paper, the first Fornasini and Marchesini (FM) model of 2D systems which is a second order recursive form is considered. Meantime, the suggested method can be simply deployed to other first or second order 2D models.
\end{abstract}

\section{INTRODUCTION}

Needless to say, a large number of ordinary phenomena in the nature have various quantities which are only functions of time and basically 1D systems can be used to represent them in mathematical language; for instance, the electrical potential of an electrical capacitor. On the other hand, there are a number of phenomena in the nature whose quantities are functions of more than one variable and necessarily none of them is time. Multidimensional systems are the mathematical framework used to describe these quantities [1]. Meantime, in industrial applications we are faced with a special form of multidimensional systems with only two independent variable called two-dimensional (2D) systems. 2D systems are used to describe a wide variety of the phenomena such as image, sound, heat, electrostatics, electrodynamics, fluid flow, or elasticity. Discrete 2D systems can be obtained by discretizing Partial Differential Equations (PDEs) [2]. To describe 2D systems like 1D systems, state space equation is commonly used. However, the method of describing 2D systems can be varied based on their applications. Besides state space equation, transfer functions and difference equations have been used so far [1].

During recent decades, multidimensional systems have been studied in many aspects and also in many applications. According to the large number of works in the literature, the stability of multidimensional systems in various models has been a matter of interest among researchers [3] - [6]. In addition, inspired by the Lyapunov stability condition, the problem of stability of $2 \mathrm{D}$ systems has been presented in [6]. Robust stability problem and also optimal guaranteed cost control of the uncertain 2D systems are considered in [7], [8] and [9].

Ahmadreza Argha, Li Li and Steven W. Su are with Faculty of Engineering and Information Technology, University of Technology, Sydney, PO Box 123, Broadway, NSW 2007, Australia.

\{Ahmadreza.Argha, Li.Li, Steven.Su\}@uts.edu.au
As an effective approach, the SMC method is used for 1D continuous and discrete time systems [10] - [12]. SMC is known as a robust control method which leads to considerable results in invariant control systems. Here, the term invariant means that the system is completely insensitive to uncertainties which are within the range space of the control input matrix. In other words, SMC provides dynamics with an invariance property to matched uncertainties [10]. Furthermore, SMC makes it possible to decouple the whole system motion to independent components with lower dimensions. Therefore, the complexity of feedback design is reduced [10]. The main idea of SMC is to force the states toward the sliding surface and remain on the sliding surface after reaching. However, chattering is a significant drawback for continuous SMC (which mainly involves a discontinuous control term). To soften the discontinuous control part, a continuous approximation is used for reducing the chattering [13]. On the other hand, for DSMC, removing the discontinuous control part from the control law leads to the elimination of the chattering issue [14], [16] and [15]. The obtained control law is called linear control law. In [17], according to the so called 1D quasi-sliding mode [12], SMC design has been extended for 2D systems in Roesser Model (RM). In addition, the conditions to ensure the remaining horizontal and vertical states in RM on the switching surfaces and also the reaching condition using a 2D Lyapunov function are investigated in [18].

As another method to deal with 2D systems, it appears that the popular strategy is to generalize $1 \mathrm{D}$ concept. Wave advance model (WAM) is a 1D form of 2D systems established by Porter-Aravena [19]. In this method, the 2D systems are considered as advanced waves and consequently the original stationary $2 \mathrm{D}$ system is converted to a time varying $1 \mathrm{D}$ system. Indeed, the state matrices have rectangular shape rather than square shape. As a result, the major drawback of this $1 \mathrm{D}$ form of 2D systems is the varying dimensions of defined state vectors. This makes it hard and probably impossible to adopt Discrete Sliding Mode Control (DSMC). Then, it is motivated to investigate another proper method to convert 2D systems to $1 \mathrm{D}$ form. In the proposed method of this paper, instead of WAM method, a row (column) processing method is used. In simpler term, 2D variables which are in the same rows (columns) form 1D stacking vectors. Consequently, the states, input and output of the obtained 1D system are in the vector form, and more importantly they have invariant dimensions. This method is basically useful for a class of 2D linear systems in which information propagation in one of the two distinct directions only occurs over a finite horizon. More 
importantly, the proposed method of this paper is effective for the classes of 2D systems which, for instance, result from certain discretization of PDEs in a spatio-temporal dynamics. This issue will be demonstrated in the next section. The 2D model considered here is FM model [20], however, our innovative technique can be deployed to other 2D models with some modifications. Eventually, after converting 2D systems to 1D form, a 1D MIMO DSMC will be designed to control original $2 \mathrm{D}$ systems.

The rest of this paper is as follows: in the next section the WAM form of 2D systems is explained and then the proposed procedure of converting a first FM model to $1 \mathrm{D}$ vectorial form is introduced. Section III describes the Discrete Sliding Mode Controller design for our problem. In Section IV, the effectiveness and efficiency of the proposed method is studied by numerical examples. Finally, Section V concludes this paper.

\section{PROBLEM FORMULATION}

To illustrate how 2D systems can be converted to $1 \mathrm{D}$ form, we consider the first FM model with the following formulation

$$
\begin{array}{r}
x(i+1, j+1)=A_{1} x(i+1, j)+A_{2} x(i, j+1)+ \\
A_{0} x(i, j)+B u(i, j)
\end{array}
$$

where $x \in R^{n}$ and $u \in R^{m}$ are respectively local state and control input. In addition, the matrices in this equation are $A_{1} \in R^{n \times n}, A_{2} \in R^{n \times n}, A_{0} \in R^{n \times n}$ and $B \in R^{n \times m}$. It can be seen that this relation is a second order recursive equation. In the rest of this section, firstly, a brief review of WAM model of 2D systems is given based on the first FM model. Secondly, the drawbacks of this method are explained. These drawbacks motivate us to investigate an alternative 1D form of 2D systems which is more effective and efficient. Finally, our proposed 1D form of first FM model is presented.

\section{A. WAM model of $2 D$ systems}

Porter and Aravena were the first investigators who considered 2D systems as a 1D model in 1984 [19]. In this model, by using a different classification on local state vectors of a 2D system, a novel form of local state vectors is achieved. In [21], it is presented that, with this new vector, study on 2D system's controllability and observability is simpler in comparison to conventional 2D methods. However, as mentioned, this proposed form has varying dimension of states, and coefficient matrices should be updated in each step. To resolve the varying dimension of state vectors in WAM model, in [19] it is suggested to expand all states to the largest dimension via augmenting system matrices with appropriate blocks of zeros. However, the system matrices are still left varying and complicated to compute. Moreover, the framework of obtaining the stack state vectors, especially for second order 2D models such as the first FM model, is relatively confusing and time-consuming. To illustrate, define the state vectors $\phi(k)$ and $v(k)$ as

$$
\begin{gathered}
\phi(k)=\left[\begin{array}{c}
x(k, 0) \\
x(k-1,1) \\
\vdots \\
x(0, k)
\end{array}\right], \\
v(k)=\left[\begin{array}{c}
u(k, 0) \\
u(k-1,1) \\
\vdots \\
u(0, k)
\end{array}\right] .
\end{gathered}
$$

In this case, the resulting WAM form of first FM model is as follows

$$
\begin{array}{r}
\phi(k+1)=M(k) \phi(k)+N(k-1) \phi(k-1)+ \\
F(k-1) v(k-1) .
\end{array}
$$

Here, matrices $M(k), N(k-1)$ and $F(k-1)$ are determined in the following forms

$$
\begin{gathered}
M(k)=\left[\begin{array}{cccc}
A_{2} & 0_{n} & \cdots & 0_{n} \\
A_{1} & A_{2} & \cdots & 0_{n} \\
0_{n} & A_{1} & \ddots & \vdots \\
\vdots & \vdots & \ddots & A_{2} \\
0_{n} & 0_{n} & \cdots & A_{1}
\end{array}\right]= \\
{\left[\begin{array}{c}
I_{k+1} \\
0_{1 \times(k+1)}
\end{array}\right] \otimes A_{2}+\left[\begin{array}{c}
0_{1 \times(k+1)} \\
I_{k+1}
\end{array}\right] \otimes A_{1},} \\
N(k-1)=T(k) \otimes A_{0}, \quad F(k-1)=T(k) \otimes B,
\end{gathered}
$$

where

$$
T(k)=\left[\begin{array}{c}
0_{1 \times k} \\
I_{k} \\
0_{1 \times k}
\end{array}\right]
$$

and $I_{k}$ is identity matrix of order $k$. According to (3) it is obvious that by defining the following relation

$$
r(k)=N(k-1) \phi(k-1)+F(k-1) v(k-1),
$$

the relation (3) is converted to a $1 \mathrm{D}$ state space model,

$$
\left[\begin{array}{l}
\phi(k+1) \\
r(k+1)
\end{array}\right]=\left[\begin{array}{ll}
M(k) & I \\
N(k) & 0
\end{array}\right]\left[\begin{array}{l}
\phi(k) \\
r(k)
\end{array}\right]+\left[\begin{array}{c}
0 \\
F(k)
\end{array}\right] v(k) .
$$

Remark 1: As can be seen, state vector in (8) is a linear combination of local states and inputs. However, in some applications, having state space equations with direct access to the local states is required. In this case, by introducing a new state vector,

$$
\begin{array}{r}
\phi(k)=[x(k+1,0), x(k, 0), x(k, 1), x(k-1,1), x(k-1,2), \\
\ldots, x(1, k-1), x(1, k), x(0, k), x(0, k+1)]^{T},
\end{array}
$$

a 1D sate space equation with direct access to the state vectors $\phi(k)$ and $\phi(k+1)$ is acquired [22].

Remark 2: In definition of state vectors (9), instead of using states just on the line $i+j=k+1$, local states located on one step before $(i+j=k)$ are also used to form state vectors. Generally, for WAM description of 2D systems which are at least second order, using state vector (9) is 
useful. However, obtaining WAM method for second order 2D systems (for instance FM model) and especially for large scale 2D systems is complicated and, more importantly, the dimension of state vector (9) is varying. On the other hand, as it will be explained in the DSMC design section, to design sliding matrices, it is necessary to find 'regular form' [28] of state space equation, while by using WAM form a new regular form should be found in each step which would result in heavy computational burden.

\section{B. New $1 D$ form of $2 D$ first FM model}

The FM model (1) can be represented in the following form

$$
\begin{aligned}
x(i+1, j+1)-A_{1} x(i+1, j) & =A_{2} x(i, j+1)+ \\
& A_{0} x(i, j)+B u(i, j) .
\end{aligned}
$$

Now, we define the following stacking vectors

$$
\begin{gathered}
V(i)=\left[\begin{array}{c}
A_{1} x(i+1,0)+A_{0} x(i, 0) \\
0 \\
\vdots \\
0
\end{array}\right], \\
X(i)=\left[\begin{array}{c}
x(i, 1) \\
x(i, 2) \\
\vdots \\
x(i, v)
\end{array}\right], U(i)=\left[\begin{array}{c}
u(i, 0) \\
u(i, 1) \\
\vdots \\
u(i, v-1)
\end{array}\right],
\end{gathered}
$$

where $v$ is the dimension of distinct variable $j, X(i) \in R^{v . n}$, $V(i) \in R^{v . n}$ and $U(i) \in R^{v . m}$. As a result, the $2 \mathrm{D}$ equation (10) can be presented as

$$
J X(i+1)=K X(i)+L U(i)+V(i),
$$

where

$$
\begin{aligned}
& J=\left[\begin{array}{cccccc}
I & 0 & 0 & \ldots & 0 & 0 \\
-A_{1} & I & 0 & \ldots & 0 & 0 \\
0 & -A_{1} & I & \ldots & 0 & 0 \\
\vdots & \vdots & & \ddots & \vdots & \vdots \\
0 & 0 & 0 & \ldots & I & 0 \\
0 & 0 & 0 & \ldots & -A_{1} & I
\end{array}\right] \\
& =I_{v} \otimes I_{n}+\left[\begin{array}{cc}
0_{1 \times(v-1)} & 0 \\
I_{v-1} & 0_{(v-1) \times 1}
\end{array}\right] \otimes\left(-A_{1}\right), \\
& K=\left[\begin{array}{cccccc}
A_{2} & 0 & 0 & \ldots & 0 & 0 \\
A_{0} & A_{2} & 0 & \ldots & 0 & 0 \\
0 & A_{0} & A_{2} & \ldots & 0 & 0 \\
\vdots & \vdots & & \ddots & \vdots & \vdots \\
0 & 0 & 0 & \ldots & A_{2} & 0 \\
0 & 0 & 0 & \ldots & A_{0} & A_{2}
\end{array}\right] \\
& =I_{v} \otimes A_{2}+\left[\begin{array}{cc}
0_{1 \times(v-1)} & 0 \\
I_{v-1} & 0_{(v-1) \times 1}
\end{array}\right] \otimes A_{0} .
\end{aligned}
$$

Besides,

$$
L=\left[\begin{array}{cccc}
B & 0 & \ldots & 0 \\
0 & B & \ldots & 0 \\
\vdots & \vdots & \ddots & \vdots \\
0 & 0 & \ldots & B
\end{array}\right]=I_{v} \otimes B
$$

Here, $x(i+1,0)$ and $x(i, 0)$ are state boundary conditions on boundary $(j=0)$. Moreover, as it is seen with the vectorial definition (11), the variable $j$ is hidden in the new defined 1D form. Model (12) is also known as descriptor model.

Remark 3: In general, the dimension of 2D systems can be infinite. However, as it was mentioned before, in this paper, it is assumed that one of the distinct variable of 2D system is finite. Moreover, the computing limitations have made it inevitable to assume finite dimensions for both separate directions of $2 \mathrm{D}$ systems. In this paper, the dimension of considered 2D system is assumed to be $\mu \times v$ and, as a result, the size of 1D state vector $X(i)$ and control input vector $U(i)$ in (12) are $v . n$ and $v . m$, respectively. Besides, there are two set of boundary conditions $(i=0$ and $j=0$ ).

$$
\left\{\begin{aligned}
\alpha(i)=x(i, 0) & \text { over } j=0, \\
\beta(j)=x(0, j) & \text { over } i=0 .
\end{aligned}\right.
$$

Remark 4: Likewise, note that matrices $J$ and $K$ are bidiagonal (in general block) Toeplitz matrices and the sizes of these matrices depend on the dimension of state vector $X(i)$. The dimensions of the matrices defined in (12) are

$$
\begin{aligned}
& J:[v \cdot n] \times[v . n], K:[v \cdot n] \times[v \cdot n], \\
& L:[v \cdot n] \times[v \cdot m] .
\end{aligned}
$$

To apply discrete sliding mode control to the system (12), this equation should be left multiplied by $J^{-1}$ (obviously, matrix $J$ is of full rank). In the case that the elements of matrix $J$ are varying, in every step the inverse of this matrix should be computed. A very heavy computational load could result, especially for 2D grids with large dimensions. However, in our case, the matrix $J$ is time invariant and consequently in the proposed DSMC of this paper, the matrix $J^{-1}$ can be computed only once.

Remark 5: In [23], a simple formula for the inverse of a block matrix with non-zero blocks in the principal diagonal and the first sub-diagonal only is proved. Adapting this formula to our case results in the following form for $J^{-1}=$ $\left[\gamma_{p, q}\right]$,

$$
\gamma_{p, q}= \begin{cases}0_{n} & \text { if } p<q, \\ I_{n} & \text { if } p=q, \\ (-1)^{p+q}\left(-A_{1}\right)^{p-q} & \text { if } p>q .\end{cases}
$$

Then, by left multiplying (12) by $J^{-1}$, the following standard 1D state space form can be obtained,

$$
X(i+1)=\hat{K} X(i)+\hat{L} U(i)+\hat{R} V(i),
$$

where

$$
\hat{K}=J^{-1} K, \hat{L}=J^{-1} L, \text { and } \hat{R}=J^{-1} \text {. }
$$

In this new 1D form, the dimension of state vectors is constant and consequently finding its regular form is possible. This sets the stage for designing specific 1D DSMC for the obtained 1D state space model (17), which is the subject of next section. 


\section{DSMC FOR 1D DISCRETE VECTOR FORM}

The SMC method has been originated from the theory of Variable Structure Systems (VSS). During recent several decades, SMC has always been considered as a powerful method for the control of systems with uncertainties. The Continuous SMC (CSMC) was primarily introduced by Utkin [10] as one of the effective nonlinear robust control approaches. It makes an invariance property to uncertainties for system dynamics. The insensitivity of the controlled system to uncertainties exists in the sliding mode, but not during the reaching phase. Owing to the pervasive usage of digital controllers, deploying SMC to discrete-time systems becomes a significant field in control theory. DSMC was introduced in the mid 80s [24]. This idea was followed by a growing list of publications [25] - [27] and also different reaching laws are proposed in [14], [16] and [29]. Similar to CSMC, the design procedure of the tracking controller is split into two steps:

1) First, sliding surface with internal stability should be designed as

$$
\sigma_{X}(i)=S X(i), \sigma_{X}(i) \in R^{v . m}
$$

where $S \in R^{[v \cdot m] \times[v \cdot n]}$.

2) Then, the control law should be designed so that states are forced to reach and stay as close as possible to the sliding surface.

Assume that $\operatorname{rank}(\hat{L})=v \cdot m$ (matrix $\hat{L}$ is of full column rank), and the pair $(\hat{K}, \hat{L})$ is controllable [27], [13].

Remark 6: Since $J$ is invertible, it is clear that the control matrix $\hat{L}$ in (17) is of full column rank if and only if the control matrix $B$ in (1) is of full column rank.

Since $\operatorname{rank}(\hat{L})=v . m$, there exists an orthogonal matrix $T_{r} \in$ $R^{[v \cdot n] \times[v \cdot n]}$ such that

$$
T_{r} \hat{L}=\left[\begin{array}{c}
0_{[v \cdot n-v \cdot m] \times[v . m]} \\
\bar{L}_{2}
\end{array}\right],
$$

where the matrix $\bar{L}_{2} \in R^{[v \cdot m] \times[v \cdot m]}$ and is nonsingular [28]. (Note that the orthogonal matrix $T_{r}$ can be computed using $Q R$ decomposition [28]). After the coordinate transformation, we have

$$
\begin{gathered}
{\left[\begin{array}{c}
Z_{1}(i+1) \\
Z_{2}(i+1)
\end{array}\right]=\left[\begin{array}{ll}
\bar{K}_{11} & \bar{K}_{12} \\
\bar{K}_{21} & \bar{K}_{22}
\end{array}\right]\left[\begin{array}{l}
Z_{1}(i) \\
Z_{2}(i)
\end{array}\right]+} \\
{\left[\begin{array}{c}
0_{[v \cdot n-v . m] \times[v . m]} \\
\bar{L}_{2}
\end{array}\right] U(i)+T_{r} \hat{R} V(i),}
\end{gathered}
$$

where

$$
\begin{gathered}
{\left[\begin{array}{l}
Z_{1}(i) \\
Z_{2}(i)
\end{array}\right]=T_{r} X(i),} \\
Z_{1}(i) \in R^{[v \cdot n-v \cdot m]} \text { and } Z_{2}(i) \in R^{v \cdot m} \\
\bar{K}_{11} \in R^{[v \cdot n-v \cdot m] \times[v \cdot n-v \cdot m]} \\
\bar{K}_{12} \in R^{[v \cdot n-v \cdot m] \times[v \cdot m]} \\
\bar{K}_{21} \in R^{[v \cdot m] \times[v \cdot n-v \cdot m]} \\
\bar{K}_{22} \in R^{[v \cdot m] \times[v \cdot m]} .
\end{gathered}
$$

This representation is referred to as 'regular form' [28]. Further, to design the sliding surface we ignore the term arising from the boundary conditions as it does not influence the stability. In these new coordinates the switching function (18) becomes

$$
\sigma_{X}(i)=\bar{S}_{1} Z_{1}(i)+\bar{S}_{2} Z_{2}(i),
$$

where $\bar{S}_{1} \in R^{[v \cdot m] \times[v \cdot n-v . m]}$ and $\bar{S}_{2} \in R^{[v \cdot m] \times[v . m]}$ satisfying $\bar{S}=$ $\left[\bar{S}_{1} \bar{S}_{2}\right]=S T_{r}^{-1}$. The design parameters $\bar{S}_{1}, \bar{S}_{2}$ determine the sliding surface and should be chosen such that, in the case that $\sigma_{X}(i)=0$, all remaining dynamics are stable. During ideal sliding on the surface, $\sigma_{X}(i)=0$ for all $k \geq k_{s}$, where $k_{s}$ is the time when sliding starts, consequently

$$
Z_{2}(i)=-\bar{S}_{2}^{-1} \bar{S}_{1} Z_{1}(i)
$$

Defining $\Omega=\bar{S}_{2}^{-1} \bar{S}_{1}$ and substituting the equation (23) into the equation (20) leads to:

$$
Z_{1}(i+1)=\left(\bar{K}_{11}-\bar{K}_{12} \Omega\right) Z_{1}(i) .
$$

As a result, stability in the sliding mode is satisfied when all eigenvalues of the matrix $\left(\bar{K}_{11}-\bar{K}_{12} \Omega\right)$ are located inside the unit circle. Indeed, the problem of finding the matrix $\Omega$ is a classical state feedback problem. In [13], it is presented that if the pair $(\hat{K}, \hat{L})$ is controllable, the pair $\left(\bar{K}_{11}, \bar{K}_{12}\right)$ is controllable as well. Therefore, any classical state feedback method can be used to compute $\Omega$. Regarding the equation (23), the matrix $\bar{S}_{2}$ plays the role of a scaling parameter which can be selected arbitrarily but invertible. For simplicity it is chosen as the following

$$
\bar{S}_{2}=\bar{L}_{2}^{-1} \text {. }
$$

With the choice (25), the matrix $\Omega$ and consequently $\bar{S}_{1}$ can be found by pole placement, LQR-design or LMI methods. In this paper the LQR-design method is used to find the matrix $\bar{S}_{1}$ by solving a certain discrete Riccati equation with proper choices of weighting matrices. In this case, the switching function can be obtained in original coordinate as in (18), where

$$
S=\bar{S}_{2}\left[\Omega \quad I_{v \cdot m}\right] T_{r}
$$

Now, to design a controller which guarantees the sliding mode of system the transformation matrix $T_{S} \in R^{[v \cdot n] \times[v \cdot n]}$ is introduced as

$$
\begin{aligned}
& T_{s}= \\
& \left.\qquad \begin{array}{cc}
I_{v \cdot n-v \cdot m} & 0_{[v \cdot n-v \cdot m] \times[v \cdot m]} \\
\bar{S}_{1} & \bar{S}_{2}
\end{array}\right] .
\end{aligned}
$$

This transformation matrix converts the system (20) to the following form:

$$
\begin{gathered}
{\left[\begin{array}{l}
Z_{1}(i+1) \\
\sigma_{X}(i+1)
\end{array}\right]=\left[\begin{array}{cc}
\tilde{K}_{11} & \tilde{K}_{12} \\
\tilde{K}_{21} & \tilde{K}_{22}
\end{array}\right]\left[\begin{array}{l}
Z_{1}(i) \\
\sigma_{X}(i)
\end{array}\right]+} \\
{\left[\begin{array}{c}
0_{[v \cdot n-v \cdot m] \times[v \cdot m]} \\
I_{v \cdot m}
\end{array}\right] U(i)+\left[\begin{array}{c}
\tilde{V}_{1} \\
\tilde{V}_{2}
\end{array}\right],}
\end{gathered}
$$

where $\left[\begin{array}{ll}\tilde{V}_{1}^{T} & \tilde{V}_{2}^{T}\end{array}\right]^{T}=T_{s} T_{r} \hat{R} V(i)$ and $\tilde{V}_{1} \in R^{[v \cdot n-v \cdot m]}, \tilde{V}_{2} \in R^{v \cdot m}$. In order to design a controller which forces the closed-loop 
system into the sliding mode we use the following linear reaching law presented in [14] and [16],

$$
\sigma_{X}(i+1)=\Phi \sigma_{X}(i)
$$

where the design parameter $\Phi \in R^{[v . m \times v . m]}$ is chosen to be a diagonal matrix with all its diagonal elements $\phi_{k}, k=$ $1, \ldots, v . m$, satisfying $0 \leq \phi_{k}<1$.

Theorem 1: Assuming the control input $U$ as:

$$
U(i)=\left[\Phi-\tilde{K}_{22}\right] \sigma_{X}(i)-\tilde{K}_{21} Z_{1}(i)-\tilde{V}_{2},
$$

the system (28) is stabilized.

Proof: Applying the above control law to the system (28) leads to the following closed-loop system:

$$
\left[\begin{array}{l}
Z_{1}(i+1) \\
\sigma_{X}(i+1)
\end{array}\right]=\left[\begin{array}{cc}
\tilde{K}_{11} & \tilde{K}_{12} \\
0 & \Phi
\end{array}\right]\left[\begin{array}{c}
Z_{1}(i) \\
\sigma_{X}(i)
\end{array}\right]+\left[\begin{array}{c}
\tilde{V}_{1} \\
0
\end{array}\right]
$$

The poles of the closed-loop system are given by

$$
\lambda\left(\mathscr{A}_{c l}\right)=\lambda\left(\tilde{K}_{11}\right) \cup \lambda(\Phi) .
$$

Obviously, the eigenvalues of $\Phi$ are assumed to be stable (by design choice). In addition, it can be easily proved that $\tilde{K}_{11}=\bar{K}_{11}-\bar{K}_{12} \Omega$ which is designed to be a stable matrix by (24). Consequently, the system (28) is stabilized with control law (30).

\section{A. Direct method to find control law}

It should be mentioned that instead of control law (30), another direct method is also possible to obtain the sliding control law [13], [15]. Assuming that matrices $\bar{S}_{1}$ and $\bar{S}_{2}$ have been designed (by for instance LQR design) such that the reduced order dynamics (24) is stable. Now, by using linear reaching law (29) we have

$$
\Phi \sigma_{X}(i)=S X(i+1) .
$$

Inserting equation (17) in (33) leads to

$$
\Phi \sigma_{X}(i)=S[\hat{K} X(i)+\hat{L} U(i)+\hat{R} V(i)] .
$$

Therefore, the control law can be defined to be

$$
U(i)=(S \hat{L})^{-1}[\Phi S X(i)-S \hat{K} X(i)-S \hat{R} V(i)] .
$$

This control law is called direct control law which can be obtained directly after computing the sliding matrix $S$.

\section{SIMULATION RESULTS}

Consider the following 2D first FM model

$$
\begin{gathered}
A_{1}=\left[\begin{array}{rr}
-0.56 & -0.33 \\
-0.10 & 0.45
\end{array}\right], A_{2}=\left[\begin{array}{ll}
0.33 & -0.54 \\
1.26 & -0.41
\end{array}\right], \\
A_{0}=\left[\begin{array}{rr}
-0.51 & -0.09 \\
0.00 & 0.04
\end{array}\right], B=\left[\begin{array}{l}
0 \\
2
\end{array}\right] .
\end{gathered}
$$

Here $x \in R^{2}$ and $u \in R$. Note that, this $2 \mathrm{D}$ system with $U=0$ is unstable. We assume this $2 \mathrm{D}$ system over the rectangle $\mu \times v(\mu=60$ and $v=39)$. Furthermore, it is supposed that

$$
\begin{gathered}
x(0, j)=\left[\begin{array}{l}
1 \\
1
\end{array}\right], \quad 0 \leq j \leq 39, \\
x(i, 0)=\left[\begin{array}{l}
0.2 \\
0.2
\end{array}\right], \quad 0 \leq i \leq 60 .
\end{gathered}
$$

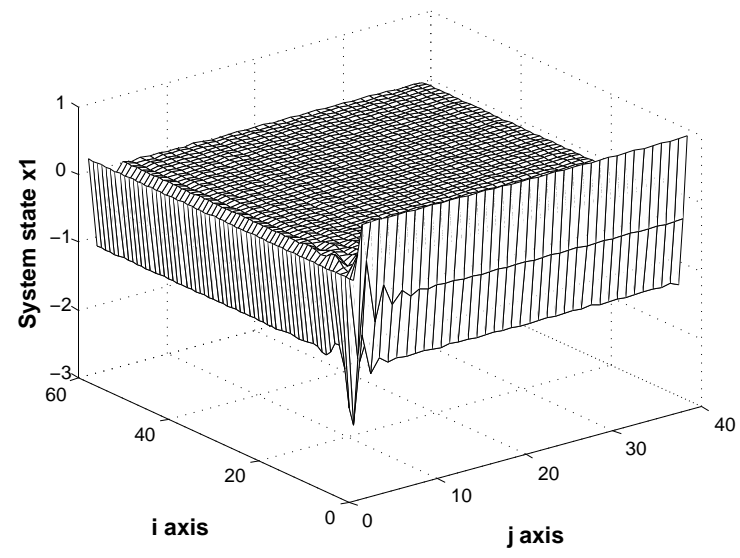

Fig. 1. The system state $x_{1}$

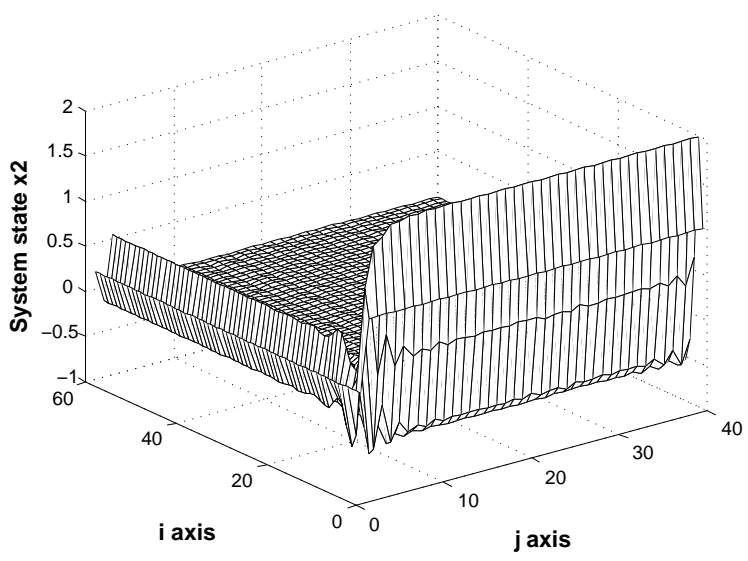

Fig. 2. The system state $x_{2}$

To compute the orthogonal matrix $T_{r}$, Matlab $Q R$ command is used. According to (25), the matrix $\bar{S}_{2} \in R^{39 \times 39}$ is chosen as $\bar{L}_{2}^{-1}$ (However, it is not necessary and can be chosen arbitrarily but invertible) and in addition matrix $\bar{S}_{1} \in R^{39 \times 39}$ is determined by LQR design with state weighting of $I_{39}$ and control weighting of $100 I_{39}$. Eventually, matrix $S$ is obtained from equation (26). $\Phi=0.5 I_{39}$ is used in control law (35) and the results of applying DSMC are given in Figures 1-3. These figures show the trajectory of 2D states $\left(x_{1}, x_{2}\right)$ and 2D control law, respectively.

\section{CONCLUSION}

In this paper we have developed a new method to apply the DSMC to the 2D first FM model using 1D vectorial form of 2D systems. Although the focus of this paper has been on the first FM model, the derived results are more general and can be easily extended to other 2D models. In the proposed 1D vectorial form of this paper, one of the 2D variables $(i$ or $j$ ) is stacked and consequently the original 2D process is replaced by a 1D virtual process which can be controlled easily. Dealing with this new 1D form, the designing procedure 


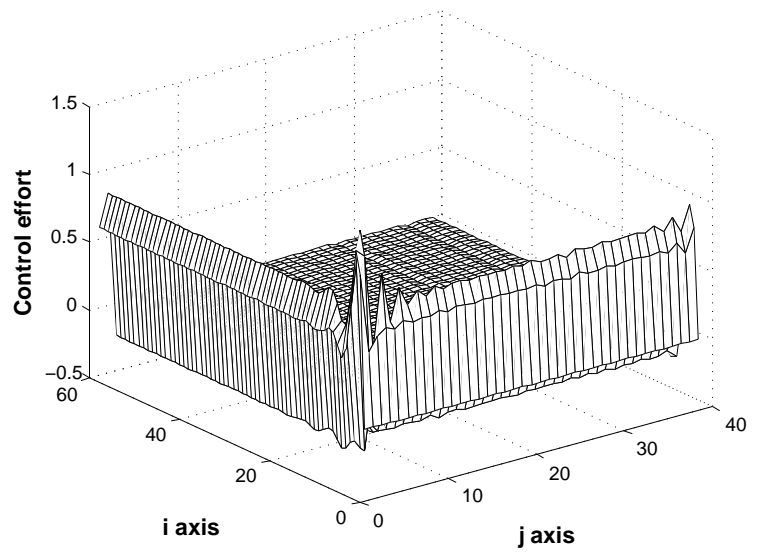

Fig. 3. The control law $u$

of DSMC is more straightforward compared to 2D system. Also, analysing the controllability of system is much easier in this form (which will be considered in the future work). Moreover, the proposed method of this paper can be extended to the tracking problem in $2 \mathrm{D}$ systems. As the future work, a new method based on a descriptor model will be investigated to avoid inverting the descriptor matrix.

\section{REFERENCES}

[1] S.G. Tzafestas, Multidimensional systems, Technique and Applications, Marcel Decker Pub., 1986.

[2] P.D. Christofides, Nonlinear and Robust Control of PDE Systems, Methods and Applications to Transport-Reaction Processes, Boston: Birkhauser, 2001.

[3] H. Kar, A new sufficient condition for the global asymptotic stability of 2D state-space digital filters with saturation arithmetic, Signal Processing Elsevier, vol. 88, 2008, pp 86-98.

[4] V. Singh, On global asymptotic stability of 2D discrete systems with state saturation, Physics Letters A Elsevier, vol. 372, 2008, pp 52875289.

[5] T. Bose, Asymptotic stability of two-dimensional digital filters under quantization, IEEE Trans. Signal Processing, vol. 42, 1994, pp 11721177.

[6] W.S. Lu, Some New Results on Stability Robustness of TwoDimensional Discrete Systems, Multidimensional Systems and Signal Processing vol. 5, 1994, pp 345-361.

[7] Z. Wang and X. Liu, Robust stability of Two-Dimensional uncertain discrete systems, IEEE Signal Processing. lett., vol. 10, 2003, pp 133136.

[8] X. Guan, C. Long and G. Duan, Robust optimal guaranteed cost control for 2D discrete systems, IEEE Proc. Control Theory and Applications, vol. 148, 2001, pp 355-361.

[9] C. Du and L. Xie, $H_{\infty}$ control and robust stabilization of twodimensional systems in Roesser models, Automatica, vol. 37, 2001, pp 205-211.

[10] V.I. Utkin, Variable structure systems with sliding modes, IEEE Trans. Automat. Contr., vol. AC-22, 1977, pp 212-222.

[11] K. Furuta, Sliding mode control of a discrete system, Syst. Contr Lett., vol. 14, 1990, pp 145-152.

[12] W. Gao, Y. Wang and A. Homaifa, Discrete-time variable structure control systems, IEEE Trans. Ind. Electron., vol. 42, 1995, pp 117122.

[13] C. Edwards, S.K. Spurgeon, Sliding Mode Control: Theory and Applications, Taylor and Francis, London, 1998.

[14] S. Hui and S. H. Zak, On discrete-time variable structure sliding mode control, Systems and Control Letters, vol. 38, 1999, pp 283-288.

[15] Monsees G. Discrete-time sliding mode control, Ph.D. Thesis, Delft University of Technology, The Netherlands, 2002.
[16] S. K. Spurgeon, Hyperplane design techniques for discrete-time variable structure control systems, International Journal on Control vol. 55(2), 1992, pp 445-456.

[17] L. Wu and H. Gao, Sliding mode control of two-dimensional systems in Roesser model, IEEE Proc. Of Control Theory and Applications, vol. 2, 2008, pp 352-364.

[18] H. Adloo, P. Karimaghaee and A.S. Sarvestani, "An Extension of Sliding Mode Control Design for the 2D systems in Roesser Model", in Joint 48th IEEE Conference on Decision and Control and 28th Chinese Control Conference Shanghai, P.R. China, Dec 2009, pp 77537758.

[19] W.A. Porter, and J.L. Aravena, 1-D model for M-D processes, IEEE, Trans. Circuits. and Systems, vol. CAS-31, NO. 8, 1984, pp 742-745.

[20] E. Fornasini and G. Marchesini, State space realization theory of 2D filters, IEEE Trans. Aut. Contr., vol. 21, 1976, pp 484-492.

[21] P.E. Wellstead and W.P. Heath, Two-dimensional control systems: Application to the CD and MD control problems, Pulp and Paper Canada vol. 95, No. 2, 1994, pp 48-51.

[22] J.L. Aravena, M. Shafiee, W.A. Porter, State models and stability for 2D filters IEEE Trans. Circuit and Systems vol. 37, No. 12, 1990, pp 1509-1519.

[23] V.N. Singh, The inverse of a certain block matrix, Bulletin of the Australian Mathematical Society, vol. 20, 1979, pp 161-163.

[24] C. Milosavljevic, General conditions for the existence of a quasisliding mode on the switching hyperplane in discrete variable structure systems, Automation and Remote Control, vol. 3, 1985, pp 36-44.

[25] H.N. Iordanou, and B.W. Surgenor, Experimental evaluation of the robustness of discrete sliding mode control versus linear quadratic control, IEEE Transactions on Control Systems Technology, vol. 5(2),1997, pp 254-260.

[26] A. Bartoszewicz, Discrete-time quasi-sliding-mode control strategies, IEEE Transactions on Industrial Electronics, vol. 45, 1998, pp 633637.

[27] G. Golo, and C. Milosavljevic, Robust discrete-time chattering free sliding mode control, Ssytems and Control Letters, vol. 41, 2000, pp $19-28$.

[28] V.I. Utkin, Sliding Modes in Control Optimization, Springer, Berlin, 1992.

[29] S.Z. Sarpturk, Y. Istefanopulos and O. Kaynak, On the stability of discrete-time sliding mode control systems, IEEE Transactions on Automatic Control vol. 32(10), 1987, pp 930-932. 